\title{
Experimental study on Electrical Resistance Tomography System for Vertical Gas/Liquid Two-phase Pipe Flow
}

\author{
Lifeng Zhang $^{\mathrm{a}}$, Qin Zhang ${ }^{\mathrm{b}}$, Feng Zhu ${ }^{\mathrm{c}}$ \\ Department of Automation, North China Electric Power University, Baoding 071003, China \\ ahdlfzhang@126.com, b714227656@qq.com, ${ }^{\mathrm{c}} 1194226905 @ q q . c o m$
}

Keywords: Gas/liquid two-phase flow, electrical resistance tomography, holdup, correlation velocity.

Abstract. Measurements of gas holdup and velocity are required in gas/liquid two-phase flow. Using electrical resistance tomography (ERT) technique, the cross-sectional image can be obtained, and hence the gas holdup is estimated. Combining ERT with the cross correlation flow measurement technique, the velocity can be calculated. A digital twin-plane ERT system for use on laboratory was developed. The velocity measurement method based on gas holdup of two plane images cross correlation was described. This paper presented the reconstructed images, gas holdup estimations and correlation velocity. Experiment results showed the feasibility of the presented method.

\section{Introduction}

Industrial flow processes are complex in nature, which often involve a variety of components in a combination of gas, liquid and solid phases. The phase distribution and interfaces in a multiphase flow carry significant information about the process. However, its behavior is extremely complex, which presents a great challenge to the study of the accurate measurement of multiphase flow [1].

Process tomography (PT) technique is a new technique that has developed rapidly in recent years and which has great potential and wide industrial application prospect for direct analysis of the characteristics of multiphase flows [2]. Electrical resistance tomography (ERT) is one kind of PT technique and has been proved to be a powerful tool for mapping the concentration and velocity distributions in two-phase flow [3, 4]. In principle, ERT can overcome the complexity of gas/liquid flow by explicitly deriving the component distributions at two adjacent planes along the pipeline. The reconstructed images can be cross-correlated to obtain the velocity profile of the flow. The non-intrusive measurement of velocity profiles within process equipment is a challenging task because few instruments can measure the velocity profile of a multi-phase flow in a pipeline. ERT provides a unique opportunity to do so by cross correlation using a twin-plane ERT sensor.

\section{Dual-plane ERT System}

Principle of ERT. A typical ERT system is composed of three parts as shown in Fig. 1: (1) the array sensor, (2) the data acquisition system (DAS) and (3) the image reconstruction and display unit. During recent decades, computer and large integrated circuits have developed rapidly.

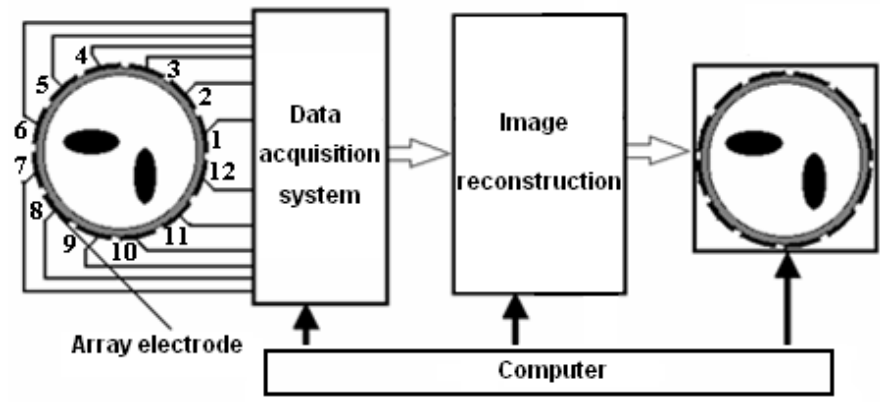

Fig. 1 Schematic diagram of ERT 
Design of the twin-plane digital ERT system. The system design followed by a modular functional design, implemented as a set of hardware circuit card units and software modules. Data from the twin-plane ERT sensor were integrated by a host PC. An architecture view for the twin-plane ERT system is shown in Fig. 2.

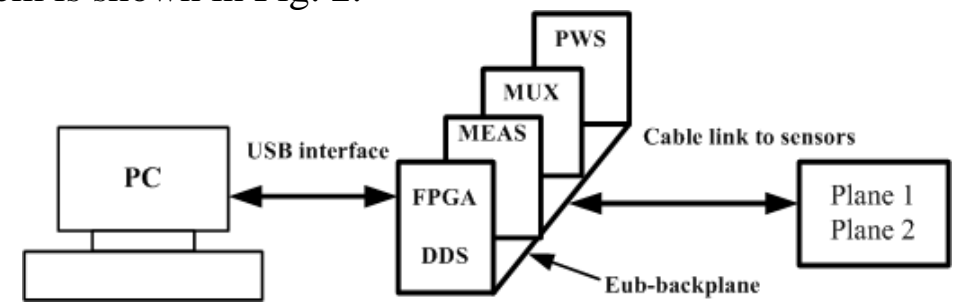

Fig. 2 ERT system platform

It includes common functions of: direct digital synthesizer (DDS) which is realized by Field-Programmable Gate Array (FPGA) and measurement (MEAS), which usually include excitation signal generation and signal condition; multiplexing (MUX), for routing exciting and measurement signals; power supply (PWS), to provide electrical power for the hardware system. The various cards are all organized using a custom-defined backplane. Control signals are provided from a FPGA chip that embraces DAS and communication functions. Data were transferred to the host PC through the Universal Serial Bus (USB) interface.

Hardware Integration of ERT. The sinusoidal current $(1 \mathrm{~Hz} 500 \mathrm{kHz}$, adjustable) excitation signal is generated by FPGA, which is sent to MEAS card to excite a pair of adjacent electrodes and the resulting potential difference is measured between another pair of adjacent electrodes to obtain a tomographic conductance projection. Measurements are routed to a 14 bit, $10 \mu$ s, sampling high precision analogue to digital converter. Resulting data are then routed via USB 2.0 interface to the host PC for further processing. The data acquisition rate of each plane is 500frames/s for twin-plane ERT sensor, while the online image reconstruction speed is 120 frames/s for each plane.

Measurement of Gas Holdup and Correlation Velocity. The gas holdup $\alpha$ can be estimated as (1)

$$
\alpha=\sum_{i=1}^{M} g_{i} A_{i} / A \text {. }
$$

Where $g_{i}$ and $A_{i}$ are the grey value and area of the $i$ th pixel, respectively. $A$ is the cross-sectional area of the pipe. $M$ is the total pixel number in cross section of the pipe.

The velocity measurement based on pixel-pixel cross-correlation has been adopted by many researchers to obtain velocity profile [5-7]. But the calculated velocity curve fluctuates very quickly based on this method. In our experiment, only the mean velocity of gas/liquid two-phase flow is needed, we introduce the cross-correlation velocity measurement method based on gas holdup of the two planes as (2)

$$
R_{k}(j \Delta)=\frac{1}{N} \sum_{i=1}^{N} \alpha_{1}(i \Delta) \alpha_{2}(i \Delta+j \Delta)
$$

Where $\alpha_{1}$ and $\alpha_{2}$ are gas holdups of the plane 1 and plane 2, respectively.

\section{Experimental Results and Analysis}

The experimental set-up is depicted in Fig. 3. It consists of the following main parts: a Perspex pipe of internal diameter $50 \mathrm{~mm}$ and length approximately $1.5 \mathrm{~m}$, twin 12-electrode sensing arrays, a TJU_ET_IV DAS system, an air compressor, four solenoids and valves, the image reconstruction computer, a flow rate meter to measure the water flow and a water pump with an inverter which can regulate the speed of the motor to control the water velocity. To simulate a kind of vertical gas/liquid flow, water is circulated via the motor and the water pump. The gas velocity is controlled through four gas valves. 


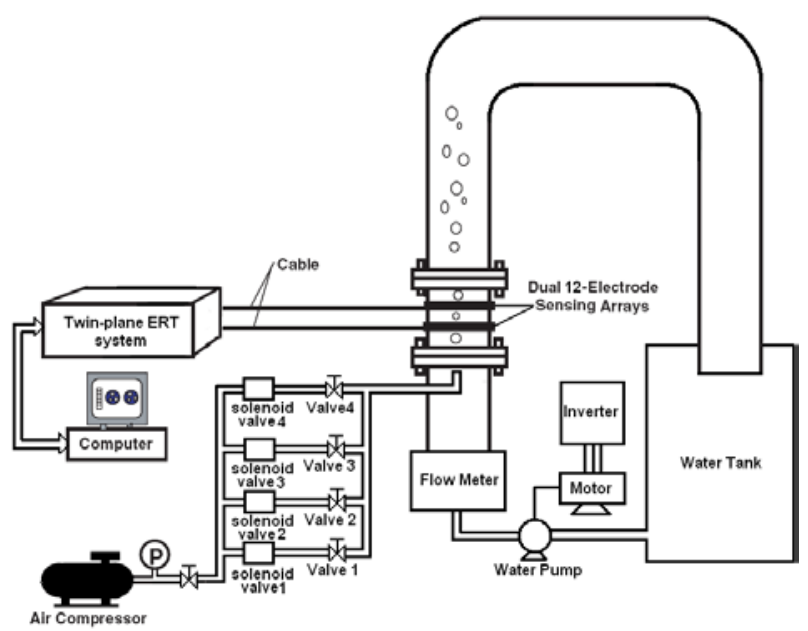

Fig. 3 Experimental set-up

Reconstructed Images and Estimation of Gas Holdup. In the experiments, the bubble flow and slug flow are tested. Fig. 4 shows the images of bubble flow and slug flow over the imaging plane at different time during their pass through the imaging cross-section. It is clearly that the temporal series of the reconstructed images can acceptably reflect the change of the cross-section distribution of the investigated two-phase flow. ERT provides a new approach to visualizing the complex motion of the bubbles in gas/water two-phase flow.

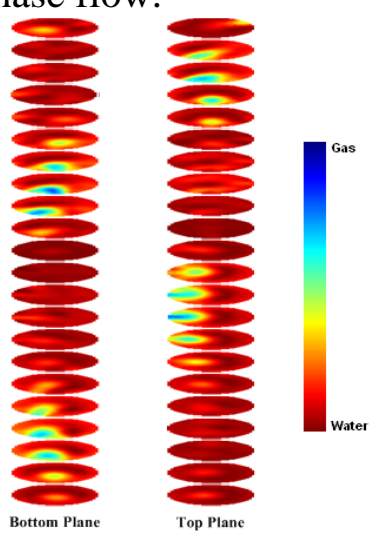

(a)

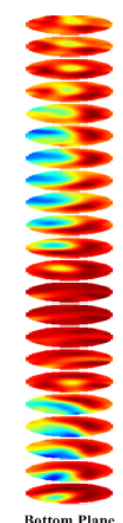

Bottom Plane

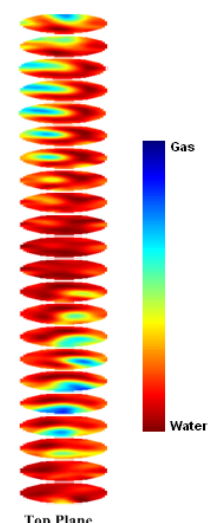

(b)

Fig. 4 Reconstructed images: (a) Bubble flow, (b) Slug flow

The gas holdup curve of each plane calculated according to (1) is shown in Fig. 5. It gives the real time estimation of gas holdup for each plane. The gas holdup curves of bottom plane and top plane are in good correlation, which were used to calculate the correlation velocity.

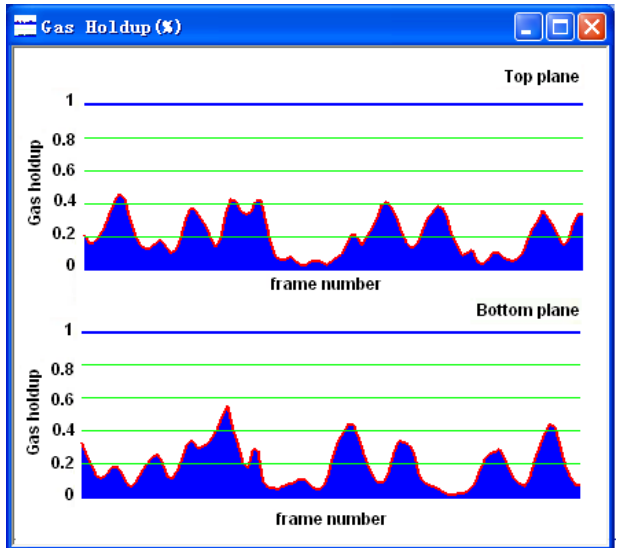

Fig. 5. The curves of gas holdup for two planes

The length of sampling images is 100, and the real time correlation velocity curve is shown in Fig. 6 . The correlation velocity is in consistent with the reconstructed images and reflects the velocity of the bubbles. 


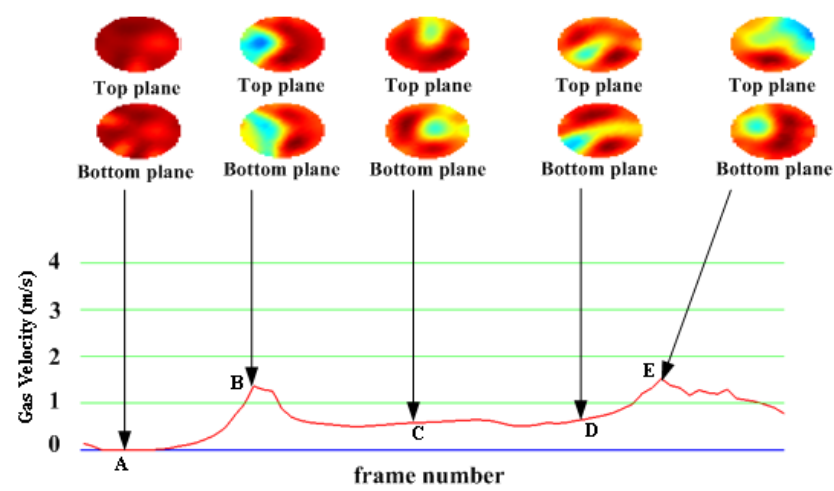

Fig. 6. Correlation velocity curve

In Fig. 6, the velocity is very low at point A when there are few bubbles. When the flow regime is slug flow, the velocity reaches a peak value at point $B$. With the decrease of bubbles, the velocity becomes lower at point $\mathrm{C}$ and $\mathrm{D}$. The velocity reaches another peak value at point $\mathrm{E}$, when the flow regime becomes slug flow again.

\section{Summary}

A digital twin-plane ERT system and the small experiment set-up were described in this pipe. Experiments results show that the reconstructed images can reflect the change of the cross-section distribution of the gas/liquid two-phase flow, the estimation of the gas holdup can be obtained, and the presented cross correlation method base on gas holdup is feasible. Further work will focus on how to improve the precision of the estimations of the gas holdup and correlation velocity.

\section{Acknowledgments}

The author wishes to thank the support of the National Natural Science Foundation of China (51306058) and the Fundamental Research Funds for the Central Universities (2014MS142).

\section{References}

[1] W. Warsito and L. -S. Fan, Measurement of real-time flow structures in gas-liquid and gas-liquid-solid flow systems using electrical capacitance tomography (ECT), Chem. Eng. Sci. 56 (2001) 6455-6462.

[2] M. Wang, A. Dorward and R. Mann, Measurement of gas-liquid mixing in a stirred vessel using electrical resistance tomography (ERT), Chem. Eng. J. 77 (2000) 93-98.

[3] H. Jiang, M. Wang and R. A. Williams, Analysis of bubble behaviors in bubble columns using electrical resistance tomography, Chem. Eng. J. 130 (2007) 179-185.

[4] Z. Y. Huang, B. L. Wang and H. Q. Li, Application of electrical capacitance tomography to the void fraction measurement of two-phase flow, IEEE. Trans. Instrum. Meas. 52 (2003) 7-12.

[5] S. Liu, Q. Chen, H. G. Wang, F. Jiang, I. Ismail and W. Q. Yang, Electrical capacitance tomography for gas-solids flow measurement for circulating fluidized beds, Flow Meas. Instrum. 16 (2005) 135-144.

[6] U. Datta, T. Dyakowski and S. Mylvaganam, Estimation of particulate velocity components in pneumatic transport using pixel based correlation with ducal plane ECT, Chem. Eng. J. 94 (2003) 87-99.

[7] V. Mosorov, D. Sanlowski, Ł. Mazurkewicz and T. Dyakowski, he 'best-correlated pixels' method for solid mass flow measurements using electrical capacitance tomography, Meas. Sci. Technol. 13 (2002) 1810-1814. 